\title{
Word Retrieval by Verbal Fluency Tasks for Young and Old People: An fNIR Study
}

\author{
In-sop Kim ${ }^{1 *}$, Natalia Jane Millin', Jaejin Hwang ${ }^{2}$ \\ ${ }^{1}$ School of Allied Health and Communicative Disorders, Northern Illinois University, DeKalb; ${ }^{2}$ Department of Industrial and Systems Engineering, \\ Northern Illinois University, DeKalb, United States
}

Purpose: There have been studies investigating the pattern of brain activation on cognitive tasks. However, at present, no study has examined verbal fluency tasks using functional near-infrared spectroscopy (fNIR) for the Korean population. The purpose of this study was to investigate age related relative changes of oxygenated hemoglobin during verbal fluency tasks using fNIR, a portable and non-invasive imaging technique.

Methods: In this study, nine young and 6 healthy older Korean individuals performed two verbal fluency tasks, a phonological task (producing words with a letter /d/) and a semantic task (producing animal category).

Results: The results showed that younger individuals performed better in the two verbal tasks and mean Hbt values, during fNIR measures, were relatively higher for younger individuals in both tasks. However, the Hbt values in the right hemisphere for older individuals were much higher than younger individuals, especially in the more difficult phonological task.

Conclusions: The results suggest that older individuals need support from the right hemisphere to successfully complete a more difficult cognitive task, unlike younger individuals. The results also suggest that brain activation during verbal fluency tasks are age related.

Keywords: Verbal fluency tasks, fNIR, Oxygenated hemoglobin, Total oxygenation (Hbt)

\section{INTRODUCTION}

One of the most commonly and frequently used cognitive tasks of executive functioning for the healthy population is a verbal fluency task. A verbal fluency task (VFT) consists of assessing word retrieval to generate as many words as possible in a set time frame. In the category fluency type (semantic), participants are instructed to generate as many words as possible for a certain category (e.g., sports, fruits or animals). In the latter fluency type (phonological), participants are instructed to produce words beginning with a given letter (e.g., A, B, D).

Verbal fluency tasks are neuropsychological in nature, activating areas of the frontal lobes and providing a measure of cognition. Successful completion of VFTs require planning and cognitive organization, abilities that are considered part of executive functioning (EF), which is controlled by the frontal lobe. Additionally, VFTs assess an individual's word knowledge and their working memory while retrieving specific words. While word knowledge is controlled by the language centers on the left hemi-

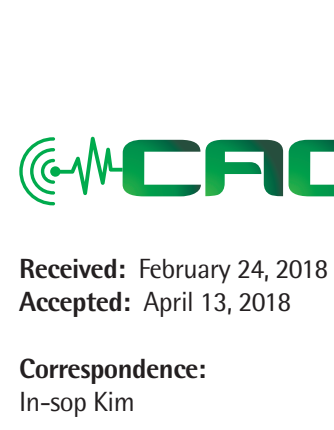

School of Allied Health and Communicative Disorders, Northern Illinois University, 354 Wirtz Hall, Northern Illinois University, DeKalb, IL, 60115, United States Tel: +815-753-7793

Fax: +815-753-9123

E-mail: ikim@niu.edu

(C) 2018 The Korean Association of SpeechLanguage Pathologists

This is an Open Access article distributed under the terms of the Creative Commons Attribution NonCommercial License (http://creativecommons.org/ licenses/by-nc/4.0/) which permits unrestricted noncommercial use, distribution, and reproduction in any medium, provided the original work is properly cited. 
sphere of the brain, the ability to eliminate words based on the demands of the task and focus only on the target (i.e., words that start with $/ \mathrm{b} /$ ) is an $\mathrm{EF}$ ability controlled by the prefrontal cortex [1]. For example, when people generate words that begin with a given letter, they will often go category by category (e.g., foods, objects) or by semantically similar words (e.g., box, boxcar, boxing).

Recent research has shown bilateral activation of prefrontal cortex during VFTs, which challenges the previous existing belief that individuals performing VFTs had left lateralized activation [2]. Lateralization research is contradictory, and more studies need to be done to reach predictable conclusions. One study found increased levels of oxygenated hemoglobin in prefrontal areas of individuals performing VFT when compared to resting baseline levels [3]. The study also measured a decrease in deoxygenated hemoglobin during VF tasks. Together, these measures demonstrate heightened prefrontal cerebral activation as a result of the tasks complex neural requirements.

Functional near-infrared spectroscopy (fNIR) has been used to measure cognitive brain activation changes [4-12]. An fNIR measures near infrared light absorbance in blood hemoglobin with and without oxygen and provides information about ongoing brain activities in a similar way to fMRI, as confirmed by several recent studies [13-20]. An fNIR provides relative concentration of oxygenated hemoglobin (Hbo), deoxygenated hemoglobin (Hbr), oxygenation (Oxy;Hbo-Hbr), and total oxygenation (Hbt). This technique overcomes many limitations of fMRI. It is portable, easy to use, and insensitive to body or head movements which can be very useful when working with children, elderly and neurogenic populations. It is also a noninvasive method for investigating brain function in real time.

Some studies have been conducted using fNIR in terms of oxygenation levels in the brain and verbal fluency tasks [2,3, 8,9,21-25]. Schecklmann et al. [9] investigated test-retest measures of brain activity during VFTs. The study investigated the feasibility of using fNIRs for test-retest measures of cognitive ability. The authors found stable behavioral data over time intervals and proved fNIR's to be a reasonable and reliable measure of brain activation during cognitive tasks over time [9]. These findings support the results from previous studies, in which brain activation was increased during VFTs.

One study investigated age related cognitive tasks with fNIR [26]. The authors found strong left lateralization in young adults during cognitive tasks. Older adults demonstrated more equal activation across the prefrontal cortex on both hemispheres. It is suggested that this highlights a neurological compensatory strategy utilized by older individuals. As certain areas of the aging brain become less effective, other regions step in to contribute to task performance. This is of interest to studies that look at age-related performance in verbal fluency tasks, since the tasks require multiple neural regions that control both semantic knowledge and executive functioning. The existing fNIR research, however, remains contradictory.

Another study investigated levels of oxygenation in prefrontal cortex during verbal fluency tasks in two different age groups [2]. The authors found reduced activation of prefrontal areas in older adults when compared to younger adults performing VFT tasks; however, despite neural activation differences, there was no significant difference in task performance across the two groups. Both older and younger adults were able to generate more words for high-productivity criteria than low-productivity criteria. It is worth noting that the older adults in the study were considered "high-performing" (i.e., well-educated), possibly affecting the results.

On the contrary, another study found that when compared to healthy aging adults, young adults outperformed their elders on VFT and word generation [23]. The study hypothesized that processing speed, not verbal abilities, explained the differences in performance. Additionally, researchers performed a study that supported previous existing research demonstrating between category performance differences across age groups [22]. In this study, younger and older adults demonstrated similar performances for VFTs (i.e., words beginning with $/ \mathrm{b} /$ ); however, younger adults significantly outperformed older adults on VF categorical tasks (i.e., fruits). Further research needs to be carried out to isolate specific performance differences between younger and older adults.

There are no studies that address the concept of verbal fluency tasks using fNIR with the Korean population. Therefore, the purpose of this study was to assess age related brain activation changes for two verbal fluency tasks. By taking individuals from different age groups, comparisons were made based on the participant's stage of life and the levels of oxygenation within the brain while performing the verbal fluency tasks.

\section{METHODS}

\section{Participants}

9 younger participants ( 5 males and 4 females, mean age: 23.9 
years old) and 6 older adults ( 3 males and 3 females, mean age: 67.7 years old) with no history of neurogenic/language disorders and psychological disorders living in South Korea, voluntarily participated in this study. The older adults performed the Korean version of the Mini-Mental Status Examination (K-MMSE) for cognitive disabilities. All of them were in the normal range (above 24). The mean score of the K-MMSE was 29 points with $0.45 \mathrm{SD}$.

\section{Procedure}

\section{Verbal fluency task}

For the phonological fluency task, each participant was asked to clearly produce as many words as possible beginning with a letter /d/ for 30 seconds. For the semantic fluency task, each participant was instructed to generate as many words as possible for an animal category for 30 seconds.

For each task, there was a 60 second break after the first task and the order of the tasks were counterbalanced.

\section{Functional near-infrared system}

The participants were monitored using functional near-infrared spectroscopy (fNIR) while accomplishing verbal fluency tasks. We used a 16-channel fNIR optical brain imaging system (fNIR 400A, Biopac company) linked to an fNIR sensor $(18 \mathrm{~cm} \times 6 \mathrm{~cm} \times 0.8 \mathrm{~cm})$ consisting of 4 LED light sources and 10 detectors. Oxygen levels in the prefrontal cortices were detected using the 16 voxels with the source detector. Therefore, 16 recording channels were generated. Cognitive optical brain imaging (COBI) studio software was used for data collection. fNIRSOFT (Biopac Systems, Inc.) visual spectroscopy signal analysis software was used to process and analyze the data [28]. The fNIR sensor, which emits light at two wavelengths $(730 \mathrm{~nm}$ and $850 \mathrm{~nm})$, was placed on the participant's forehead over the international (10-20) electrode EEG placement F7, Fp1, Fp2, and F8 (left and right hemispheres) positions and secured using a flexible headband [27]. A baseline was established by asking the participants to close their eyes for approximately 1 minute before the verbal fluency tasks took place. fNIR data was collected while the participants were instructed to generate as many animal names or nouns with an initial letter /d/ as possible.

\section{fNIR data processing}

The relative concentrations of average values for oxygenated hemoglobin (Hbo) and total oxygenation ( $\mathrm{Hbt}$ ) in the prefrontal cortex, including Brodmann's areas 10, 9, 45, and 46, were collected and used as the dependent variable in this study. The average changes in relative concentrations of oxygenation comparing to the initial eye close phase were calculated using modified Beer-Lambert Law. The raw light intensity measures were filtered using the low pass filter with a cut off at $0.1 \mathrm{~Hz}$, and motion artifacts, induced by the head or body movement, were removed by using a sliding window motion artifact rejection (SMAR) system within fNIRSOFT. The exported data was further analyzed using Matlab ${ }^{\circledR 2017 a}$ (The MathWorks Inc., Natick, USA). The fNIR Hbo and Hbt data was analyzed for both hemispheres.

\section{RESULTS}

\section{Verbal fluency tasks}

The mean numbers of naming words with a letter /d/ (phonological fluency task) for younger and older participants were 6.7 and 4.2. Also, the mean numbers of naming animals (semantic fluency task) for younger and older groups were 13 and 9.9 respectively.

The one-way ANOVA results showed that there was a significant difference in the task of naming words with a letter /d/ for younger and older groups, $F(1,13)=8.83, p<0.05$, However, there is no difference in the task of naming animals between the two groups, $F(1,13)=0.771, p=0.391$ (Figure 1).

\section{fNIR Analysis}

A $2 \times 2 \times 2$ analysis of variance (ANOVA) mixed design with a between factor (younger, older) and two tasks (letter and animal naming), and hemisphere (left and right) as within factors for the mean Hbo values did not show any significant main effects on the three independent variables and interac-

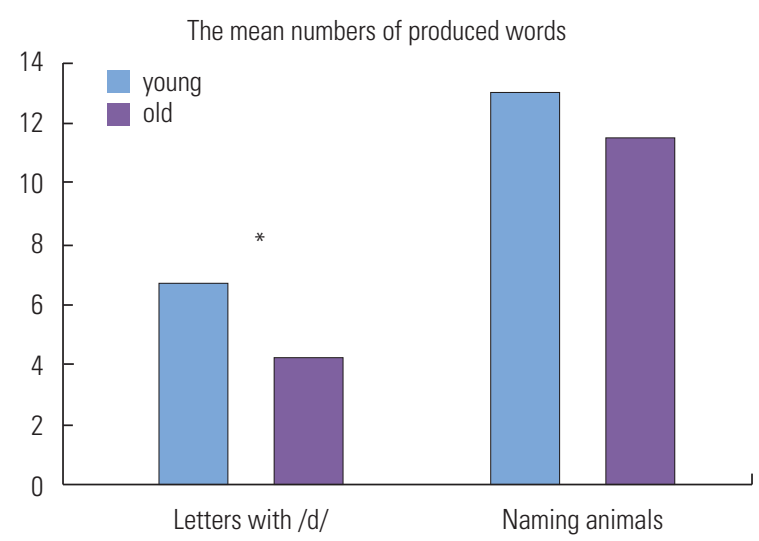

Figure 1. The mean numbers of word production for verbal fluency tasks. * Significant difference $p<0.05$. 
tion effects among them. Therefore, the mean Hbt values for the three variables were further analyzed. All effects were reported as significant at $p<0.05$. There was a significant main effect on hemisphere, $F(1,52)=6.37$ indicating that there is the mean Hbt value difference between left $(\mathrm{m}=1.2)$ and right $(m=1.96)$ hemispheres. There was also an interaction effect between age and hemisphere, $F(1,52)=9.98$. This indicates that the mean Hbt value differences on left and right hemispheres differ in younger and older groups. Following a simple effect analysis revealed that the older group showed the significant Hbt difference between the left and the right hemisphere, $F(1,56)=11.20$. Furthermore, after analyzing the interaction graph, mean $\mathrm{Hbt}$ values of the right hemisphere were higher than that of the left hemisphere in the older group (Figure 2).

There was a significant interaction effect between tasks and age, $F(1,52)=4.84$. This indicates that the mean Hbt differences between the two tasks differ in younger and older groups. A simple effect analysis was performed comparing

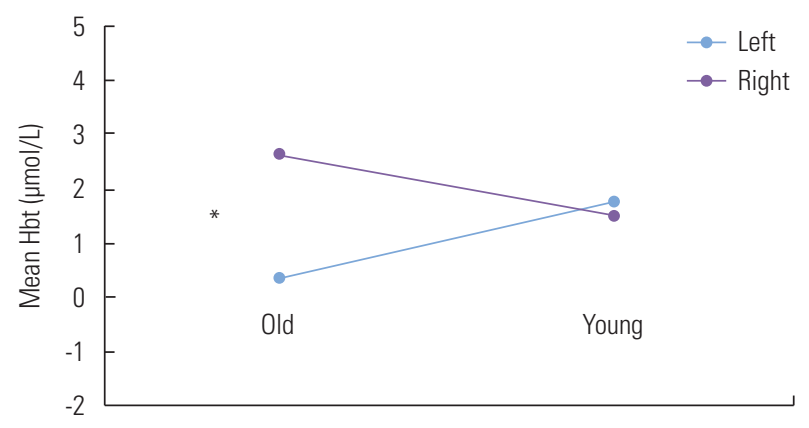

Figure 2. The mean $\mathrm{Hbt}$ of each hemisphere across younger and older groups. *Significant difference $p<0.05$.

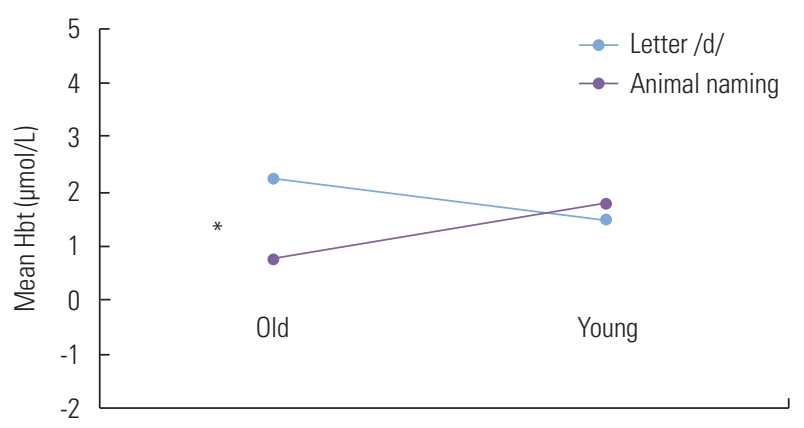

Figure 3. The mean $\mathrm{Hbt}$ of each task across younger and older groups. ${ }^{*}$ Significant difference $p<0.05$. each task to each age group. This revealed a significant interaction when comparing the mean Hbt of older and younger to the letter $/ \mathrm{d} /$ naming task compared to the animal naming task $F(1,56)=4.19$. There was a significant mean Hbt difference in the older group. Furthermore, the interaction graph shows that the older group had a higher Hbt mean value in the letter / $\mathrm{d} /$ naming task than in the animal naming task (Figure 3).

Finally, the age $\times$ hemisphere $\times$ tasks interaction was significant, $F(1,52)=7.30$. This indicates that previously described interaction of hemispheres $\times$ age differ in the letter $/ \mathrm{d} /$ and the animal naming tasks.

The result of following analysis showed that the mean $\mathrm{Hbt}$ difference between the left and right hemisphere in the older group was much larger than in the younger group at the letter $/ \mathrm{d} /$ naming task, $F(1,26)=15.9$. There was no significant mean Hbt difference of hemispheres comparing to the two age groups at the animal naming task, $F(1,26)<1$ (Figure 4).

Another analysis of simple main effect revealed that the

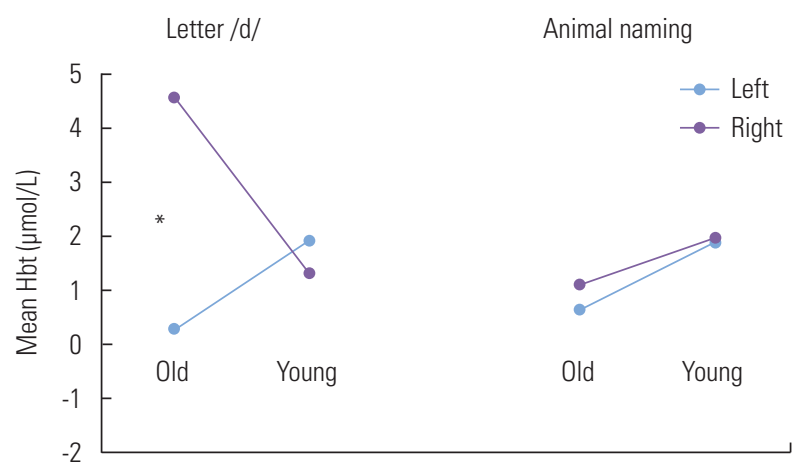

Figure 4. The mean Hbt difference between left and right hemispheres of younger and older groups across the two tasks.

${ }^{*}$ Significant difference $p<0.05$.

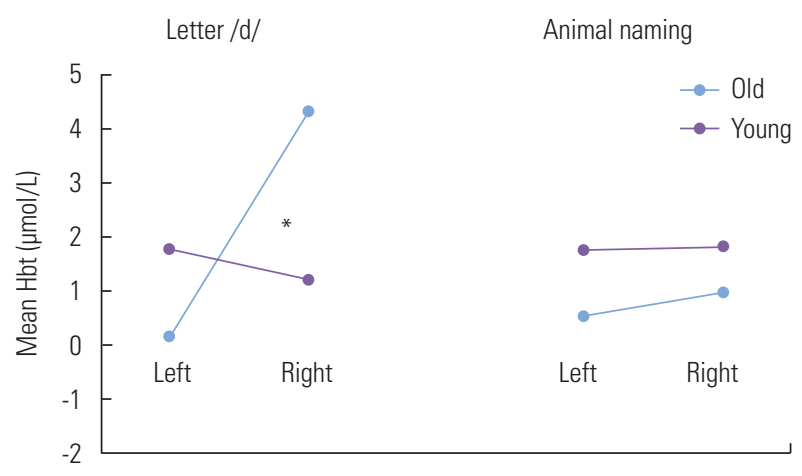

Figure 5. The mean Hbt difference between younger and older groups at left and right hemispheres across the two tasks.

*Significant difference $p<0.05$. 
mean Hbt value difference between younger and older groups in the right hemisphere was larger than in the left hemisphere at the letter $/ \mathrm{d} /$ naming task, $F(1,26)=22.4$. There was no significant difference in the animal task, $F(1,26)<1$ (Figure 5).

\section{DISCUSSION AND CONCLUSIONS}

The aim of the current study was to investigate the concentrations of oxygenated hemoglobin and total hemoglobin levels for two different verbal fluency tasks in young and elderly Korean participants. We examined Hbo and Hbt values across two verbal fluency tasks for younger and older groups. For the behavioral test, all participants produced fewer words in the letter naming task than in the animal naming task. The study revealed a significant difference in behavioral results for the letter naming task, but no significant difference was found in the animal naming task. This indicates that the phonological fluency task (letter /d/ naming) is more difficult than the semantic fluency task (animal naming) for both younger and older groups. The older group also showed more difficulty in the more challenging task (letter /d/ naming). The results in the current study are not consistent with some of the previous findings, in which older participants performed as well as the younger participants $[2,21]$. However, this may be explained by a verbal fluency study [2], in which the authors concluded that the comparable performance was due to highly educated older participants. Older participants in our study, unlike in the previous study, were not highly educated.

The fNIR brain activation results showed that there was a significant Hbt difference between left and right hemispheres for the older group, but no significant difference for the younger group. The results were consistent across tasks. This suggests that the younger group used left and right hemisphere more equally regardless of how difficult the tasks were.

Furthermore, the older group showed significant $\mathrm{Hbt}$ value differences between two verbal fluency tasks. However, there were no Hbt value differences between the two verbal fluency tasks for the younger group. Specifically, when the older group performed the letter /d/ naming task, Hbt values of the left and right hemispheres were significantly different. Hbt values on the right hemisphere were much higher than on the left hemisphere in the older group. However, this phenomenon did not occur when they performed on the animal naming task. This suggests that when a task is more difficult, the older group may need more involvement of right hemisphere to deal with the task. On the contrary, the younger group possi- bly can manage a difficult task with relatively equal levels of Hbt activation on both hemispheres.

The results of the current study demonstrate a consistent pattern, in which the younger participants show higher oxygenated hemoglobin levels than the older participants in both the left and right hemispheres during the animal naming tasks, but not during the letter /d/ tasks. These results are consistent with those in some of the previous related fNIR studies $[2,5,22]$ They found a constant decrease in oxygenation levels with healthy elderly participants. According to Kahlaoui et al. [2], significant reduced oxygenated hemoglobin was observed in elderly participants than in younger participants for both letter and category verbal fluency tasks.

However, in the current study during the letter naming task there was an exception for the brain activation pattern, which showed higher activation in right hemisphere in older participants compared to younger participants. This might be somewhat explained by the phenomenon described as Hemispheric Asymmetry Reduction in Older Adults (HAROLD) [26]. This theory suggests that prefrontal brain activity is more lateralized in the left hemisphere in younger participants than in older participants, during cognitive activities while older participants show more bilateral activation. In our study, the younger participants showed higher levels of $\mathrm{Hbt}$ in the left hemisphere compared to the right hemisphere during the letter naming task. Relatively the same pattern was shown during animal naming tasks. However, the older participants showed significantly higher Hbt levels in the right hemisphere during the letter naming task. Thus, consistent with younger participants and inconsistent with older participants in this theory. The reasons for the Hbt asymmetry in the older participants are obscure. One possibility is that older participants need extra support from the right hemisphere to complete the task. Therefore, in a relatively difficult cognitive task, brain reorganization occurs to compensate for the reduced prefrontal activation $[2,21,26,29]$.

Some limitations of this study should be addressed. First, a larger group of younger and older individuals could influence the results. Furthermore, a gender effect could be explored with a larger participant group. Second, only one type of letter condition (d) and category condition (animal) was utilized to examine verbal fluency in this study. Using different types of verbal fluency tasks could bring about more meaningful results. Third, the fNIR tool used in the study itself has a limitation since it covers limited brain areas. It would be beneficial to use full head fNIR which records hemodynamic informa- 
tion from more cerebral cortices to be able to investigate meaningful brain activation in verbal fluency tasks. Therefore, these limitations need to be addressed in future studies.

In conclusion, this study investigated age related brain activation changes for phonological and semantic verbal fluency tasks. The behavioral results showed that younger individuals produced more words for both tasks. We also found a significant word production difference for the phonological verbal fluency task between the two groups. The fNIR results showed that older individuals used the right hemisphere more significantly to complete the phonological verbal tasks. Younger individuals showed higher activation in both hemispheres during the semantic verbal task, but not in the phonological fluency task, compared to the older individuals. These results suggest that there are age related brain activation differences during verbal fluency tasks.

\section{ACKNOWLEDGEMENTS}

The authors presented some portions of the data at the 2016 American Speech Hearing Association (ASHA) convention, Philadelphia, Pennsylvania.

\section{REFERENCES}

1. Murray L, Clark H. Neurogenic disorders of language and cognition. 2nd ed. Austin, TX: Pro-ed; 2015.

2. Kahlaoui K, Di Sante G, Barbeau J, Mheux M, Lesage F, Ska B, et al. Contribution of NIRS to the study of prefrontal cortex for verbal fluency in aging. Brain Lang. 2012;121(2):164-173.

3. Herrmann MJ, Ehlis AC, Fallgatter AJ. Frontal activation during a verbal-fluency task as measured by near-infrared spectroscopy. Brain Res Bull. 2003;61:51-56.

4. Kito H, Ryokawa A, Kinoshita Y, Sasayama D, Sugiyama N, Ogihara T, et al. Comparison of alterations in cerebral hemoglobin oxygenation in late life depression and Alzheimer's disease as assessed by near-infrared spectroscopy. Behav Brain Funct. 2014; 10(1):1-9.

5. Tsujii T, Okada M, Watanabe S. Effects of aging on hemispheric asymmetry in inferior frontal cortex activity during belief-bias syllogistic reasoning: a near infrared spectroscopy study. Behav Brain Res. 2010;210(2):178-183.

6. Hock C, Müller-Spahn F, Schuh-Hofer S, Hofmann M, Dirnagl U, Villringer A. Age dependency of changes in cerebral hemoglobin oxygenation during brain activation: a near-infrared spectroscopy study. J Cereb Blood Flow and Metab. 1995;15:1103-1108.

7. Mehagnoul-Schipper DJ, van der Kallen BF, Colier WN, van der Sluijs MC, van Erning LJ, Thijssen HO, et al. Simultaneous measurements of cerebral oxygenation changes during brain activa- tion by near-infrared spectroscopy and functional magnetic resonance imaging in healthy young and elderly participants. Hum Brain Mapp. 2002;6:14-23.

8. Sakatani K, Lichty W, Xie Y, Li S, Zuo H. Effects of aging on language activated cerebral blood oxygenation changes of the left prefrontal cortex: near infrared spectroscopy study. J Stroke Cerebrovasc Dis. 1999;8:398-403.

9. Schecklmann M, Ehlis AC, Plichta M, Fallgatter AJ. Functional near-infrared spectroscopy: a long-term reliable tool for measuring brain activity during verbal fluency. Neuroimage. 2008;43(1): 147-155.

10. Schroeter ML, Zysset S, Kruggel F, von Cramon DY. Age dependency of the hemodynamic response as measured by functional near-infrared spectroscopy. Neuroimage. 2003;19:555-564.

11. Tuscan LA, Herbert JD, Forman EM, Juarascio AS, Izzetoglu M, Schultheis MT. Exploring frontal asymmetry using functional near-infrared spectroscopy: a preliminary study of the effects of social anxiety during interaction and performance tasks. Brain Imaging Behav. 2013;7:140-153.

12. Watanabe A, Matsuo K, Kato T. Cerebrovascular responses to cognitive tasks and hyperventilation measured by multi-channel near-infrared spectroscopy. J Neuropsychiatry Clin Neurosci. 2003;15:442-449.

13. Ayaz H, Izzetoglu M, Platek SM, Bunce S, Izzetoglu K, Pourrezaei K, et al. Registering fNIR data to brain surface image using MRI templates. IEEE Trans Radiat Plasma Med Sci. 2006;1:4906-4909.

14. Cui X, Bray S, Bryant DM, Glover GH, Reiss AL. A quantitative comparison of NIRS and fMRI across multiple cognitive tasks. Neuroimage. 2011;54:2808 -2821.

15. Irani F, Platek SM, Bunce S, Ruocco AC, Chute D. Functional Near Infrared Spectroscopy (fNIRS): an Emerging Neuroimaging Technology with Important Applications for the Study of Brain Disorders. Clin Neuropsychol. 2007;21:9-37.

16. Izzetoglu M, Bunce SC, Izzetoglu K, Onaral B, Pourrezaei K. Functional brain imaging using near-infrared technology. IEEE Trans Radiat Plasma Med Sci. 2007;26:8-46.

17. Kennan RP, Kim D, Maki A, Koizumi H, Constable RT. Non-invasive assessment of language lateralization by transcranial near infrared optical topography and functional MRI. Hum Brain Mapp. 2002;16:183-189.

18. Plichta MM, Herrmann MJ, Baehne CG, Ehlis AC, Richter MM, Pauli P, et al. Event-related functional near-infrared spectroscopy (fNIRS): are the measurements reliable? Neuroimage. 2006;31: 116-124.

19. Plichta MM, Herrmann MJ, Baehne CG, Ehlis AC, Richter MM, Pauli P, et al. Event-related functional near-infrared spectroscopy (fNIRS) based on craniocerebral correlations: reproducibility of activation? Hum Brain Mapp. 2007;28:733-741.

20. Toronov V, Webb A, Choi JH, Wolf M, Michalos A, Gratton E, et al. Investigation of human brain hemodynamics by simultaneous near-infrared spectroscopy and functional magnetic resonance imaging. Int J Med Phys Clin Eng Radiat Oncol. 2001;28:521.

21. Cabeza R, Anderson ND, Locantore JK, McIntosh AR. Aging 
gracefully: compensatory brain activity in high-performing older adults. Neuroimage. 2002;17:1394-1402.

22. Herrmann MJ, Walter A, Ehlis AC, Fallagatter AJ. Cerebral oxygenation changes in the prefrontal cortex: effects of age and gender. Neurobiol Aging. 2006;27:880-894.

23. McDowd J, Hoffman L, Rozek E, Lyons KE, Pahwa R, Burns J, et al. Understanding verbal fluency in healthy aging, Alzheimer's disease, and Parkinson's disease. Appl Neuropsychol Adult. 2011;25 (2):210-225.

24. Kameyama M, Fukuda M, Uehara T, Mikuni M. Sex and age dependencies of cerebral blood volume changes during cognitive activations: a multichannel near-infrared spectroscopy study. Neuroimage. 2004;22:1715-1721.

25. Kono T, Matsuo K, Tsunashima K, Kasai K, Takizawa R, Rogers MA, et al. Multiple-time replicability of near-infrared spectrosco- py recording during prefrontal activation task in healthy men. Neurosci Res. 2007;57:504-512.

26. Cabeza R. Hemispheric asymmetry reduction in older adults: The HAROLD model. Psychol Aging. 2002;17:85-100.

27. Homan RW, Herman J, Purdy P. Cerebral location of international 10-20 system electrode placement. Electroencephalogr Clin Neurophysiol Suppl. 1987;66(4):376-382.

28. Ayaz H, Izzetoglu M, Shewokis PA, Onaral B. Sliding-window motion artifact rejection for functional near-infrared spectroscopy. Proc Int Conf Biomed Eng Inform. 2010;6567-6570.

29. Vermeij A, van Beek AHEA, Olde Rikkert MGM, Claassen JAHR, Kessels RPC. Effects of aging on cerebral oxygenation during working-memory performance: a Functional Near-Infrared Spectroscopy Study. PLoS ONE [Internet]. 2012 [cited 2018 Jan 20];7(9): 6210. 